Int. J. Morphol.,

33(3):1093-1101, 2015.

\title{
Perfil Antropométrico por Posición de Juego en Handbolistas Chilenos
}

\author{
Anthropometric Profile in Chilean Handball Players According to Playing Position
}

Barraza, F.*; Yáñez, R.**; Tuesta, M.***; Núñez, P.**; Zamora, Y.** \& Rosales, G.*****

BARRAZA, F.; YÁÑEZ, R.; TUESTA, M.; NUÑEZ, P.; ZAMORA, Y. \& ROSALES, G. Perfil antropométrico por posición de juego en handbolistas chilenos. Int. J. Morphol., 33(3):1093-1101, 2015.

RESUMEN: El objetivo fundamental de la presente investigación fue determinar el perfil antropométrico y su relación con la capacidad de salto en jugadores de balonmano varones de la región de Valparaíso y de la Selección Chilena, esto en relación a su posición en el campo de juego. Se valoraron 74 jugadores de la categoría cadetes, cuyas edades fluctuaron entre los 14 y los 16 años, pertenecientes a los equipos: Club Italiano Villa Alemana, Club Italiano Quillota, Club Luterano, Club Viña del Mar, Club San José y Selección Chilena. Los resultados arrojaron que en cuanto a las variables antropométricas, la Selección Chilena y el Club Italiano Villa Alemana, son los que poseen los parámetros que más se asemejan a los requeridos a nivel competitivo dentro de esta categoría. Al hacer referencia a las variables antropométricas se estimó que, en todos los grupos estudiados, la masa adiposa se relacionó negativamente con la altura de salto alcanzada. Mientras que la masa muscular presentó una alta correlación con el rendimiento en salto en la mayoría de los equipos. Los índices de correlación evidenciaron que un menor porcentaje de masa grasa y mayor porcentaje de masa muscular favorecen la altura de salto alcanzada en todas las posiciones de juego. Queda de manifiesto que la relación masa adiposa y masa muscular es un indicador importante a la hora de determinar el rendimiento en la capacidad de salto en deportistas con características similares a las de este estudio.

PALABRAS CLAVE: Perfil antropométrico; Composición corporal; Somatotipo; Handbolistas; Capacidad de salto; Balonmano.

\section{INTRODUCCIÓN}

El deporte de elite es cada vez más exigente y competitivo por lo que se requiere que los atletas se encuentren en la mejor forma deportiva. Es por este motivo que se busca en los jóvenes deportistas de clubes y selecciones características antropométricas que permitan una mejora en el rendimiento deportivo. Aunque resulta obvio que el entrenamiento físico juega un papel fundamental en la consecución del éxito deportivo, la realidad, es que no existen procedimientos capaces de modificar de manera significativa los límites impuestos por la naturaleza. Diversos estudios han demostrado que, a igualdad de condiciones de entrenamiento físico, tanto desde el punto de vista cuantitativo como cualitativo, los mejores resultados deportivos, corresponden a aquellos sujetos con unas condiciones anatómicas más favorecedoras para la práctica del deporte en cuestión, considerando las características antropométricas parte del conjunto de variables biológicas relacionadas con el rendimiento deportivo (Esparza, 1993).
Considerando esto, el balonmano no se queda atrás, ya que cada vez son más las demandas y exigencias morfológicas que deben tener los jóvenes deportistas para alcanzar un máximo rendimiento y así soportar las diversas exigencias que requiere este deporte. En este contexto Bayer (1987), señala que "para el desarrollo óptimo de los deportistas es necesario que se trabajen los aspectos técnicos, tácticos, psicológicos y antropométricos". Con este último factor se puede dar respuesta a las diversas exigencias que se exhiben en las innumerables situaciones presentes en una competencia. Las capacidades físicas que los atletas de este deporte deben desarrollar son: la fuerza, velocidad, resistencia y potencia. La potenciación de estas capacidades físicas, en las que los aspectos antropométricos son determinantes, permite superar las diferentes situaciones de la realidad de juego. Por otro lado los factores que condicionan el alto rendimiento en balonmano son: la antropometría, la condición física, la técnica, la táctica y los aspectos psicológicos de cada deportista (Moreno, 2004).

Escuela de Educación, Carrera Educación Física. Universidad Viña del Mar, Viña del Mar, Chile.

** Facultad de Filosofía y Educación, Pontificia Universidad Católica de Valparaíso, Valparaíso, Chile.

*** Carrera de Kinesiología, Universidad Santo Tomas, Viña del Mar, Chile.

***** Centro tecnológico de investigación y gestión en actividad física, deporte y salud, Valparaíso, Chile. 
En el balonmano, como en otros deportes, la posición en el campo de juego es de real importancia debido a la función que cada jugador debe cumplir dentro del campo de juego, función condicionada por características antropométricas del jugador.

En el jugador de Handball de alto rendimiento, existe un gran desarrollo de la resistencia aeróbica, pero principalmente se evidencia una gran predominancia del metabolismo anaeróbico aláctico, aspectos que son relevantes para la ejecución motriz en este deporte (Van Muijen et al., 1991). En los handbolistas de elite se aprecian elevados valores de la fuerza máxima, de la potencia muscular de extremidades inferiores y superiores, asociados a una gran velocidad de lanzamiento, factores importantes en el rendimiento de los jugadores dentro del campo de juego (Gorostiaga et al., 1999), sin embargo, surge la interrogante de la real importancia que adquieren los parámetros antropométricos para el desarrollo de la saltabilidad como una forma de establecer mejoras en el rendimiento deportivo de los jugadores, lo cual podría influir de manera favorable en el aumento de la potencia muscular. En este marco, el presente estudio tuvo por objetivo la determinación del perfil antropométrico y su relación con el grado de saltabilidad de jugadores de balonmano cadetes varones de la región de Valparaíso y de la Selección Chilena sub-16, según su posición en el campo de juego, se consideró que por ser jugadores de clubes y selección, ya presentan un cierto grado de especialización en cuanto a la posición que ocupan en el campo de juego. Finalmente, por medio de este estudio se determinó la influencia de las variables antropométricas en el desarrollo de la capacidad de salto y a su vez permitió establecer criterios de selección y de distribución por posición en el campo de juego, en jugadores Chilenos de Handball.

\section{MATERIAL Y MÉTODO}

En el presente estudio fueron evaluados 74 adolescentes Handbolistas de sexo masculino, de la categoría cadete (entre 14 y 16 años), pertenecientes a equipos de la región de Valparaíso y el equipo 2013 de la Selección Chilena de la misma categoría. Actualmente sus planteles de honor participan en torneos a nivel nacional e internacional.

Las evaluaciones se realizaron a sujetos que pertenecían a 6 equipos de Balonmano de la región de Valparaíso, a saber: Club Italiano de Villa Alemana $(\mathrm{n}=14)$, Club Italiano Quillota ( $n=13)$, San José ( $n=11)$, Luterano $(n=8)$, Club Viña $(n=7)$ y Selección Chilena $(n=21)$. La distribución por posición de juego fue la siguiente; Porteros $(n=9)$, Pri- mera línea (n=37), Extremos (n=19), Pivotes (n=9). Los datos de medidas básicas por club se observan en la Tabla I.

La evaluación antropométrica se realizó antes de cada entrenamiento en los mismos lugares de entrenamiento de cada equipo y consideró la utilización de equipo deportivo liviano y el vaciado urinario. Se midió bajo el protocolo de marcaje de la International Society for the Advancement of Kineantropometriy (ISAK) para el procedimiento de medición de 25 variables perfil restringido descrito por Drinkwater(1984) y Norton \& Olds (1996). Para la determinación de la composición corporal, se consideró el tejido adiposo, muscular, óseo, residual y piel, utilizando las ecuaciones propuestas por Kerr (1988). Se determinó la forma corporal de los sujetos a través del método del somatotipo de Carter (Carter \& Heath, 1990; Carter, 2002).

Para el procedimiento de evaluación se utilizó el kit antropométrico Rosscraft, el cual está compuesto por los instrumentos: antropómetro largo, antropómetro corto, segmómetro, calibrador de pliegues cutáneos o plicómetro, cinta métrica para perímetros, un estadiómetro para estatura y una balanza CAM para la medición del peso corporal con precisión de $100 \mathrm{~g}$. Se evaluaron 3 variables básicas: peso, estatura de pie, estatura sentado; 6 diámetros óseos, (biacromial, humeral, tórax anteroposterior, tórax transverso, biepicondilar); 10 perímetros (cabeza, brazo contraído, brazo relajado, antebrazo máximo, tórax mesoesternal, cintura, cadera, muslo máximo, muslo medio, pantorrilla), 6 pliegues cutáneos (tríceps, subescapular, supraespinal, abdominal, muslo medio y pantorrilla), se evalúo también la velocidad y altura de salto alcanzada. A partir de estas variables se estimó, la composición corporal y el somatotipo (Tabla I).

Para estimar la altura alcanzada y la velocidad de salto se utilizó una alfombra de contacto Axon Jump modelo T.

\section{RESULTADOS}

Se consideró preciso entregar un análisis completo de los resultados, con la finalidad de poder establecer las reales incidencias y relaciones entre las diferentes variables de estudio. En la Tabla I se pueden apreciar los resultados de las variables antropométricas, de la altura alcanzada y la velocidad de salto de los deportistas distribuidos por equipos, los datos muestran la Media y desviación estándar.

En la Tabla II, se resumen los resultados de las variables antropométricas y de la altura y velocidad de salto de los deportistas distribuidos por posición de juego, al igual que en la Tabla I, se visualiza la media y la desviación estándar. 
BARRAZA, F.; YÁÑEZ, R.; TUESTA, M.; NUÑEZ, P.; ZAMORA, Y. \& ROSALES, G. Perfil antropométrico por posición de juego en handbolistas chilenos. Int. J. Morphol., 33(3):1093-1101, 2015.

Tabla I. Promedios, desviaciones estándar y niveles de significancia de las variables: Peso, talla, perímetro de brazo contraído, perímetro de antebrazo, perímetro de muslo medio, perímetro de pierna, diámetro biacromial, masa adiposa, masa muscular, sumatoria de 6 pliegues, endomorfía, mesomorfía, ectomorfía, altura y velocidad alcanzada en salto vertical de los equipos evaluados.

\begin{tabular}{|c|c|c|c|c|c|c|c|c|c|c|c|c|}
\hline \multirow[b]{2}{*}{ Club } & \multicolumn{2}{|c|}{$\begin{array}{c}\text { C. Italiano, } Q \text {. } \\
(n=13)\end{array}$} & \multicolumn{2}{|c|}{$\begin{array}{c}\text { C. San José, C } \\
(n=11)\end{array}$} & \multicolumn{2}{|c|}{$\begin{array}{c}\text { C. Luterano } \\
\text { C. }(n=8)\end{array}$} & \multicolumn{2}{|c|}{$\begin{array}{c}\text { C. Italiano } \\
\text { V.A }(n=14)\end{array}$} & \multicolumn{2}{|c|}{$\begin{array}{l}\text { C. Viña del } \\
\text { Mar }(n=7)\end{array}$} & \multicolumn{2}{|c|}{$\begin{array}{c}\text { Selección Chilena } \\
(n=21)\end{array}$} \\
\hline & Media & DE & Media & DE & Media & DE & Media & DE & Media & DE & Media & DE \\
\hline Peso & 71,0 & 17,6 & 72,7 & 10,5 & 72,9 & 14,1 & 65,4 & 7,4 & 68,7 & 15,0 & 73,9 & 10,1 \\
\hline Talla & 172,7 & 6,3 & 171,8 & 5,1 & 173,8 & 9,6 & 173,6 & 8,7 & 173,8 & 8,4 & 178,3 & 3,8 \\
\hline P. Brazo contraído & 29,8 & 3,3 & 30,7 & 1,8 & 31,0 & 2,8 & 29,8 & 1,8 & 28,3 & 3,4 & 31,5 & 2,1 \\
\hline P. Antebrazo & 25,3 & 2,0 & 25,8 & 1,2 & 26,0 & 2,1 & 25,5 & 1,4 & 24,4 & 1,9 & 26,6 & 3,1 \\
\hline P. Muslo medio & 52,5 & 8,6 & 52,1 & 3,5 & 50,6 & 3,5 & 50,2 & 3,1 & 49,9 & 5,9 & 52,0 & 3,5 \\
\hline P. Pierna & 37,2 & 4,0 & 36,9 & 2,6 & 37,4 & 3,9 & 35,5 & 1,8 & 35,3 & 3,1 & 36,2 & 2,5 \\
\hline D. Biacromial & 38,8 & 2,3 & 39,1 & 2,0 & 39,8 & 3,0 & 38,6 & 1,7 & 37,7 & 2,4 & 40,0 & 1,8 \\
\hline Masa Adiposa (\%) & $30,8^{*}$ & 4,9 & 28,9 & 5,0 & 28,6 & 3,1 & 27,3 & 3,9 & 29,0 & 4,5 & $25,3^{*}$ & 3,4 \\
\hline Masa Muscular (\%) & $41,6^{*}$ & 4,4 & 43,8 & 3,6 & 43,1 & 2,2 & 43,7 & 3,0 & 43,1 & 2,1 & $45,6^{*}$ & 1,9 \\
\hline$\sum 6$ pliegues & $94,2^{*}$ & 46,3 & 92,7 & 46,2 & 90,3 & 27,0 & 64,7 & 20,8 & 72,4 & 31,7 & $60,8^{*}$ & 24,6 \\
\hline Endo & $4,2 *$ & 2,1 & 4,3 & 2,1 & 4,2 & 1,3 & 3,0 & 0,9 & 2,9 & 1,3 & $2,8^{*}$ & 1,2 \\
\hline Meso & 4,8 & 1,5 & 4,9 & 1,2 & 4,9 & 1,0 & 4,3 & 1,0 & 3,9 & 0,9 & 4,3 & 0,9 \\
\hline Ecto & 2,5 & 1,7 & 1,9 & 1,0 & 2,1 & 1,0 & 3,0 & 1,2 & 2,7 & 1,3 & 2,7 & 1,1 \\
\hline Altura Alcanzada & $35,4 * *$ & 5,4 & $33,8^{* *}$ & 7,4 & 36,2 & 4,2 & 38,2 & 5,0 & 36,5 & 8,2 & $42,0^{* *}$ & 4,6 \\
\hline Velocidad $(\mathrm{m} / \mathrm{s})$ & 2,6 & 0,2 & 2,6 & 0,3 & 2,7 & 0,2 & 5,4 & 9,9 & 2,7 & 0,3 & 2,9 & 0,2 \\
\hline
\end{tabular}

(*) Diferencias significativas con una p $<0,05$ en prueba T, entre los grupos. (**) Diferencias significativas con una $p<0,05$ en prueba $T$, entre los grupos.

Tabla II. Promedios, desviaciones estándar y niveles de significancia de las variables: Peso, talla, perímetro de brazo contraído, perímetro de antebrazo, perímetro de muslo medio, perímetro de pierna, diámetro biacromial, masa adiposa, masa muscular, sumatoria de 6 pliegues, endomorfía, mesomorfía y ectomorfía, altura y velocidad alcanzada en salto vertical por posición de juego.

\begin{tabular}{|c|c|c|c|c|c|c|c|c|}
\hline \multirow[b]{2}{*}{ Posición } & \multicolumn{2}{|c|}{$\begin{array}{c}\text { Porteros } \\
(n=9)\end{array}$} & \multicolumn{2}{|c|}{$\begin{array}{c}\text { P. Línea } \\
(\mathrm{n}=37)\end{array}$} & \multicolumn{2}{|c|}{$\begin{array}{c}\text { Extremo } \\
(n=19)\end{array}$} & \multicolumn{2}{|c|}{$\begin{array}{l}\text { Pivote } \\
(n=9)\end{array}$} \\
\hline & Media & $\mathbf{D E}$ & Media & DE & Media & DE & Media & DE \\
\hline Peso & $86,3^{*}$ & 15,4 & 68,7 & 8,0 & 61,8 & 5,2 & $84,7^{*}$ & 11,3 \\
\hline Talla & 177,1 & 3,2 & 175,7 & 6,9 & $169,5^{*}$ & 4,9 & 177,7 & 8,8 \\
\hline P. Brazo contraído & $32,5^{*}$ & 2,7 & 30,0 & 2,2 & 28,9 & 1,9 & $33,2 *$ & 2,2 \\
\hline P. Antebrazo & $28,4^{*}$ & 3,9 & 25,7 & 1,5 & 24,4 & 1,4 & $26,7^{*}$ & 1,4 \\
\hline P. Muslo medio & $56,0^{*}$ & 4,0 & 50,2 & 2,9 & 48,8 & 3,1 & $57,7^{*}$ & 7,5 \\
\hline P. Pierna & $39,3^{*}$ & 2,7 & 35,8 & 2,1 & 34,8 & 2,3 & $39,5^{*}$ & 3,6 \\
\hline D. Biacromial & 39,2 & 2,7 & 39,2 & 1,9 & $38,3 * *$ & 1,9 & $40,8^{* *}$ & 2,8 \\
\hline Masa Adiposa & 30,5 & 2,7 & 27,8 & 4,6 & 26,1 & 3,9 & 30,1 & 5,1 \\
\hline Masa Muscular & 42,6 & 2,8 & 44,0 & 3,5 & 44,4 & 3,1 & 42,8 & 3,3 \\
\hline$\sum 6$ pliegues & $110,6^{*}$ & 40,8 & 69,0 & 25,9 & 60,3 & 24,1 & $109,5^{*}$ & 47,5 \\
\hline Endo & $4,9 *$ & 1,5 & 3,1 & 1,3 & 2,7 & 1,2 & $5,1^{*}$ & 2,1 \\
\hline Meso & $5,5^{*}$ & 1,1 & 4,3 & 1,0 & 4,2 & 0,9 & $5,3^{*}$ & 1,2 \\
\hline Ecto & $1,2 *$ & 0,7 & 2,9 & 1,2 & 2,9 & 1,0 & $1,5^{*}$ & 1,1 \\
\hline Altura Alcanzada & 35,0 & 5,9 & $39,1 * *$ & 6,1 & 38,8 & 4,5 & $32,2 * *$ & 7,0 \\
\hline Velocidad (m/s) & 2,6 & 0,2 & 3,7 & 6,0 & 2,8 & 0,2 & 2,5 & 0,3 \\
\hline
\end{tabular}

La Tabla III, muestra los índices de correlación de las variables peso, masa adiposa, masa muscular y altura alcanzada por posición de juego.

Para estimar si existían diferencias significativas entre los grupos evaluados se utilizó la prueba de comparacio- nes múltiples de Scheffé corroborando que existieron diferencias significativas entre los grupos y las variables mencionadas $(\mathrm{p}<0,05)$.

En la tabla I, se pueden apreciar diferencias significativas en las variables masa adiposa, masa muscular, 


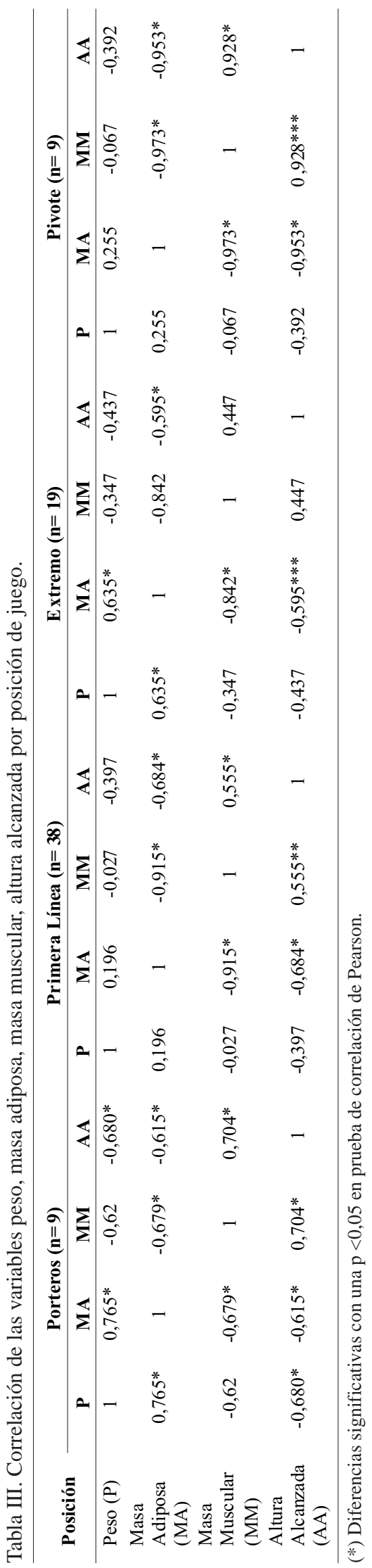

1096 sumatoria de pliegues y componente endomórfico entre el Club Italiano de Quillota y la selección Chilena. También se pueden apreciar diferencias significativas entre la selección Chilena y los Clubes Italiano de Quillota y San José en lo que concierne a la altura de salto alcanzada.

En la Tabla II, se aprecian diferencias significativas en las variables peso, perímetro de brazo contraído, perímetro de antebrazo, perímetro muslo medial, perímetro de pierna, suma de seis pliegues y componentes endomórfico, mesomórfico y ectomórfico al realizar la comparación en las posiciones de juego, porteros y pivotes contra los primera línea y extremos. También se evidenció una estatura inferior en la posición extremos la cual es significativa. También se presentaron diferencias significativas en la altura alcanzada de salto entre las posiciones primera línea y pivotes $(\mathrm{p}<0,05)$. Los porteros presentaron un somatotipo promedio meso endomorfo, los primera línea un somatotipo mesomorfo balanceado, los extremos un somatotipo mesomorfo balanceado y los pivotes un somatotipo meso endomorfo.

En la Tabla III, se puede apreciar que la variable peso solo se asoció negativamente con altura de salto en porteros, mientras que en todos los grupos la masa adiposa se relacionó negativamente con la altura de salto alcanzada $(\mathrm{p}<0,05)$. También se evidenció que la masa muscular se correlacionó de manera positiva con la altura de salto alcanzada en las posiciones portero, primera línea y pivote $(\mathrm{p}<0,05)$, no ocurriendo esto en la posición extremos.

En la Figura 1, se aprecia que los porteros y los pivotes presentan un peso signficativamente mayor que los primera línea y los extremos $(\mathrm{p}<0,05)$.

En la Figura 2 se aprecia una gran dispersión de los datos en lo que respecta a los clubes evaluados en este estudio, se evidenció la mayor distrubución de los puntos en la zona central de la somatocarta, esto demostró una tendencia a la mesomorfia en este grupo de deportistas.

En la Figura 3 se aprecia la dispersión de los datos en lo que respecta las posiciones de juego. Se evidencia una predominante tendencia a la mesomorfia.

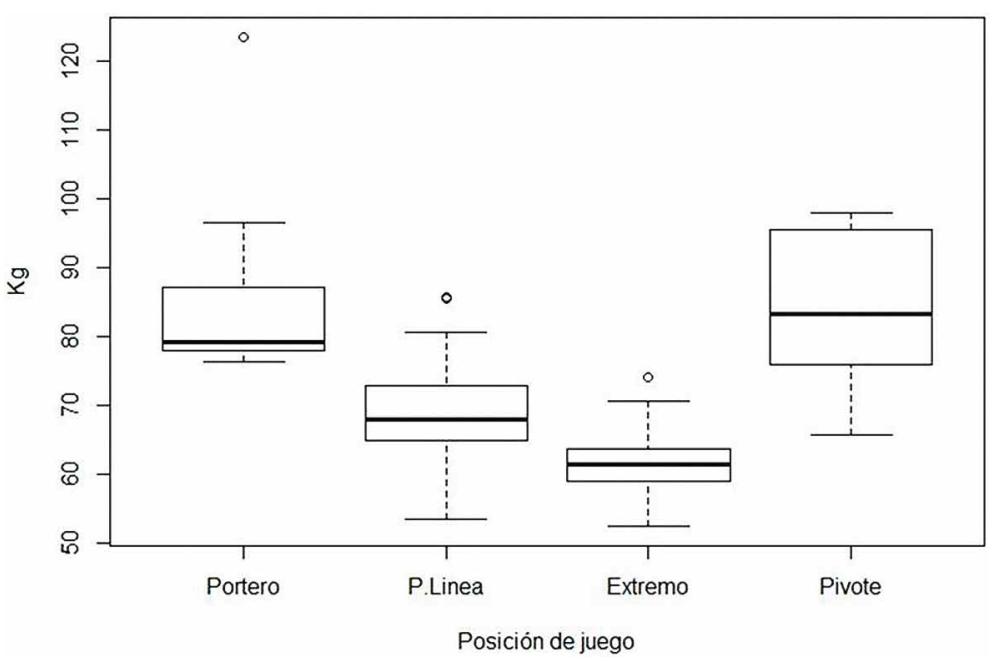

Fig. 1. Comparación del peso según la posición de juego. 


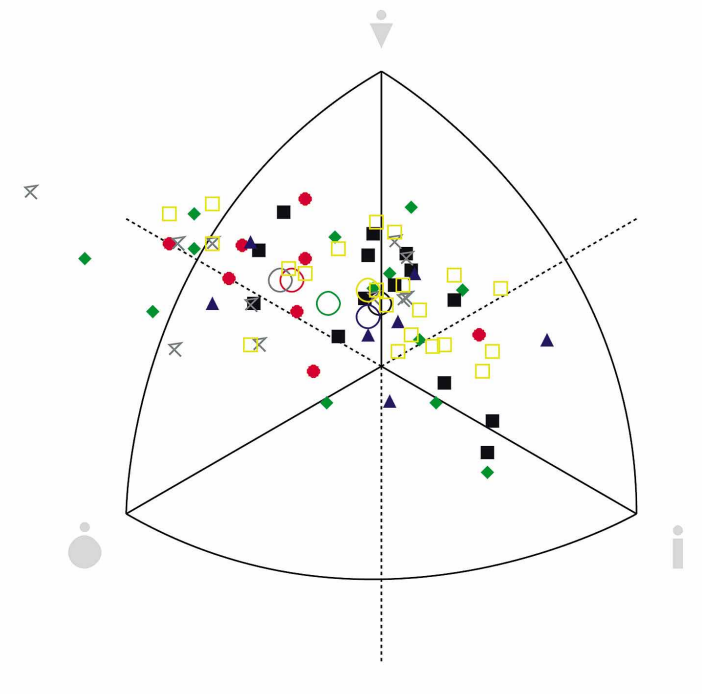

\begin{tabular}{|l|l|l|}
\hline$\bullet$ Club Italiano de Quillota & $\square$ Club Italiano & $\bullet$ Club Luterano \\
\hline$又 \quad$ Club San José & $\Delta$ Club Viña & Selección Chilena \\
\hline
\end{tabular}

Fig. 2. Dispersión de los puntos en la somatocarta de los clubes evaluados.

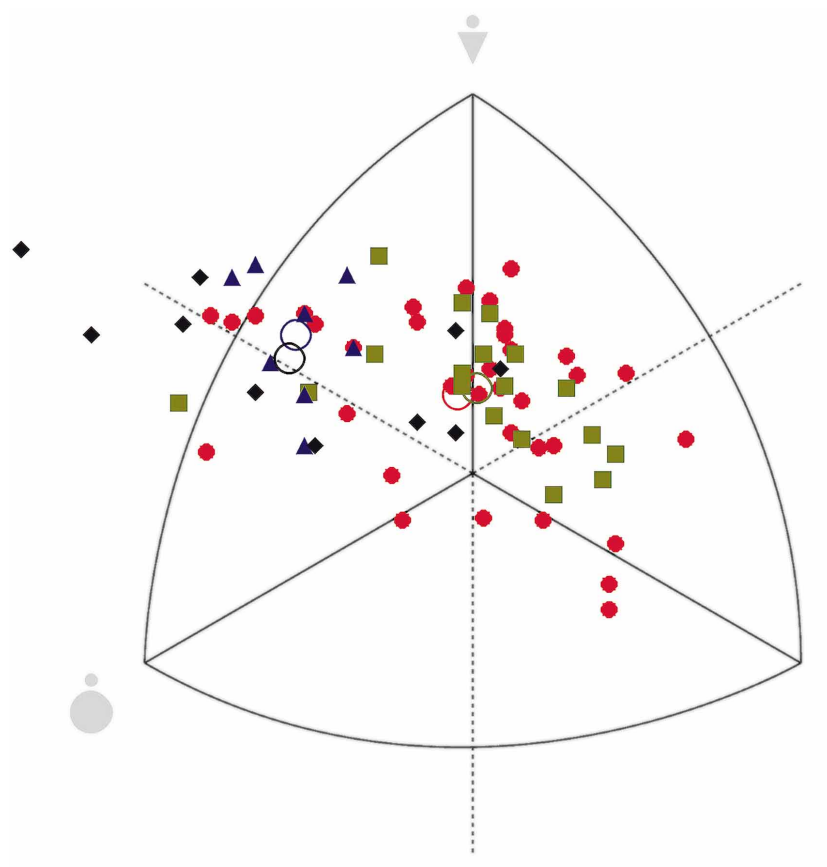

\begin{tabular}{|l|l|}
\hline Primera Línea & $\square$ Extremo \\
\hline Pivote & $\Delta$ Porteros \\
\hline
\end{tabular}

Fig. 3. Dispersión de los puntos en la somatocarta de los sujetos por posición de juego.

\section{DISCUSIÓN}

La valoración de la estructura corporal a partir de variables antropométricas es un aspecto fundamental en la evaluación integral de un deportista (Norton \& Olds). Tanto las variables de capacidad física como las relacionadas con la valoración antropométrica, composición corporal y somatotipo, desempeñan un papel muy importante y fundamental en todos los niveles de competición (Srhoj et al., 2002). Al contrastar los datos del perfil antropométricos de la variable peso con los resultados obtenidos por Fernández (2000) en su estudio realizado en jóvenes deportistas de España, se pudo apreciar que la media obtenida de $69,3 \mathrm{~kg}$ fue similar a la encontrada en la investigación realizada, ya que los valores promedio de los clubes estudiados oscilaron entre los 65,4 y 73,9 $\mathrm{kg}$. Respecto a la variable estatura, el estudio español obtuvo una media de $176,6 \mathrm{~cm}$, en el caso de los clubes chilenos estos evidenciaron un promedio de $173,0 \mathrm{~cm}$.

Analizando los resultados por clubes, se obtuvo que en la variable masa muscular la selección Chilena, el club San José y el club Italiano Villa Alemana obtuvieron los resultados más elevados, con porcentajes de $45,6 \%, 43,8 \%$ y $43,7 \%$ respectivamente. Los resultados obtenidos en este estudio demuestran que a mayor porcentaje de masa adiposa mayor valor de peso corporal $(\mathrm{p}<0,05)$. En esta investigación se evidenció que en todas las posiciones de juego, se da que a mayor porcentaje de masa adiposa, menor es la altura de salto alcanzada $(p<0,05)$. Esto deja de manifiesto que el aumento de masa adiposa es un condicionante negativo en el rendimiento deportivo, específicamente en la altura de salto alcanzada.

En relación a la capacidad de salto obtenido por posición de juego, se considera que la capacidad más importante que deben poseer los jugadores que realizan deportes colectivos, es la capacidad de resistir en el tiempo a manifestaciones de fuerza dinámica (Ekblom, 1986), los resultados muestran la tendencia en estos equipos, de que a menor peso corporal, mayor masa muscular y menor masa adiposa, por ende, mayor altura alcanzada en el salto. Desglosando los datos por equipo, fue posible señalar que la Selección Chilena y el Club Italiano Villa Alemana presentaron los mejores resultados, alcanzando un promedio de $42,0 \mathrm{~cm}$ y $38,2 \mathrm{~cm}$ respectivamente. Resulta necesario mencionar además que la Selección Chilena presenta el menor porcentaje de masa grasa y el mayor porcentaje de masa muscular. Este mismo grupo obtiene la mejor altura de salto, confirmando la relación positiva entre porcentaje de masa muscular y altura alcanzada. Esto dejó de manifiesto que la relación masa 
adiposa y masa muscular es un indicador importante a la hora de determinar el rendimiento en la capacidad de salto en deportistas con características similares a las de este estudio. Con respecto a la capacidad de salto, se puede afirmar que las capacidades de condición física menos modificables por el entrenamiento son las relacionadas con la velocidad: siendo a la vez éstas las que más se relacionan con la eficacia en el rendimiento en balonmano (velocidad de desplazamiento, velocidad en las acciones, capacidad de salto, capacidad de lanzamiento, etc) (Moreno, 2004).

Respecto al somatotipo de los equipos, estos presentaron una tendencia a la mesomorfia. Los resultados obtenidos en un estudio realizado en Murcia en jugadores juveniles de Handball confirman los resultados obtenidos en este estudio ya que señalan que estos deportistas poseen un somatotipo con tendencia a la mesomorfia (Vila et al., 2009).

Al referirnos a las características antropométricas por posición de juego se evidencia que los porteros poseen un alto valor respecto al peso obtenido $(86,3 \mathrm{~kg})$ en comparación con el estudio de Ramírez et al. (2006), quien evaluó a jugadores de balonmano de Portugal, obteniendo una media de $76,75 \mathrm{~kg}$. Pese a la diferencia de los resultados, sigue la tendencia del juego actual, en el que predomina un alto valor de peso en los porteros. Lo cual se complementa con un alto valor en el porcentaje de masa adiposa y además un menor valor de masa muscular. Los porteros y pivotes obtienen los menores valores en la altura alcanzada $(35,0 \mathrm{~cm}$ y $32,2 \mathrm{~cm}$ respectivamente) y velocidad de salto $(2,6 \mathrm{~m} / \mathrm{s})$. Entonces, se puede estimar que la variable masa adiposa influye negativamente en la capacidad de salto. El estudio de Malina \& Bouchard (1991), demostró que el salto vertical tuvo una alta correlación con el sprint. También se confirmó una alta correlación entre la fuerza y el sprint, en los porteros esta capacidad es de vital importancia debido a su rol y acciones llevadas a cabo en el campo de juego.

En cuanto a las características de los primeras líneas se observa que respecto al perfil antropométrico, la variable peso arroja que los primera línea (centrales y laterales) poseen uno de los menores promedios $(68,5 \mathrm{~kg})$, ubicándose sólo por sobre los extremos. Cabe señalar que estos datos concordaron con las tendencias detectadas por Vila et al. donde se señala que los centrales junto a los extremos son los que poseen menor peso por el hecho de que los centrales necesitan saltar más y ser más ligeros para ejecutar acciones veloces. Tal como se evidencia en los datos obtenidos en la variable estatura, estos poseen el segundo valor más bajo $(175,7 \mathrm{~cm})$, ubicándose bajo el nivel de los extremos. Respecto a los porcentajes de masa muscular, se pudo evidenciar que poseen el segundo mayor valor (44,0 \%), lo que indica un alto nivel de masa muscular y además un bajo valor de masa adiposa con respecto a las demás posiciones de juego. El elevado valor de la masa muscular para este grupo se ve reflejado en la capacidad de salto, evidenciando un promedio de $(39,1 \mathrm{~cm})$ de altura alcanzada, reflejando los mayores valores en la altura alcanzada, lo que se relaciona con el mejor resultado obtenido en la capacidad de salto. Al momento de clasificar a los primera línea por su somatotipo, estos se encuentran en la categoría mesomorfo balanceado $(3,1-4,3-2,9)$.

Respecto a los extremos, los datos obtenidos permitieron apreciar un peso de $61,8 \mathrm{~kg}$ y una talla de $169,5 \mathrm{~cm}$. El porcentaje de la masa muscular obtenida fue la más elevada con respecto a las demás posiciones de juego, con un promedio de $44,4 \%$. Además, en este grupo se evidenció un menor valor en la masa adiposa con un promedio de $26,1 \%$ confirmando la tendencia que se da en este estudio, que indica que a mayor masa muscular, menor masa adiposa y por consiguiente mayores valores en la capacidad de salto. Los altos valores de masa muscular se ven reflejados en el somatotipo, clasificándose en la categoría mesomorfo balanceado $(2,7-4,2-2,9)$, lo cual indica que estos sujetos poseen mayor volumen muscular, huesos y articulaciones de mayores dimensiones.

Los pivotes, por su parte, presentaron un promedio de $84,7 \mathrm{~kg}$, lo que resultó menor al mostrado por Ramírez et al. (2006) quienes señalaron que la media obtenida en la variable peso de los jugadores cadetes de Portugal, fue de $87 \mathrm{~kg}$. En lo que respecta a la estatura, los pivotes alcanzaron el promedio más alto $(177,7 \mathrm{~cm})$. Esta medida, en comparación con el estudio de Ramírez et al., presentó un valor menor. En la variable masa muscular se obtuvo un promedio de $42,8 \%$, siendo el segundo grupo que obtuvo el menor registro. Además, presentaron una mayor sumatoria de pliegues en comparación con los primera línea y extremos, siendo estas significativas. No ocurrió lo mismo en la posición de porteros, lo que se tradujo finalmente en menor capacidad de salto en estos sujetos por posición de juego. Complementando lo anteriormente expuesto, los pivotes en las variables de perímetros, obtuvieron los valores más elevados de perímetro muslo medio $(57,7 \mathrm{~cm})$ y pierna $(39,5 \mathrm{~cm})$, lo que se correlaciona de forma negativa con la capacidad de salto, ya que, según los resultados obtenidos en este grupo a mayor perímetro del miembro inferior, muslo y pierna, se asoció en este caso, con menor masa muscular total y mayor nivel de masa adiposa, se obtuvo una menor altura alcanzada en la prueba de salto $(32,2 \mathrm{~cm})$. Así se corrobora la idea de que los pivotes, según sus características de juego, no requieren de un gran desarrollo de la saltabilidad. Dentro de la clasificación del somatotipo, los pivotes presentan un componente mesomorfo que sobresale de los demás componentes, clasificándose como mesoendomorfos $(5,1-5,3-1,5)$. 
En virtud de lo expuesto anteriormente, se pudo evidenciar que dentro de las variables antropométricas estudiadas la masa muscular se correlaciona de manera positiva con la capacidad de salto, esto queda demostrado en las posiciones portero, primera línea y pivotes más no así en la posición extremos. En el caso de la masa adiposa, los resultados demostraron que en todas las posiciones de juego los mayores valores de adiposidad reflejan un menor rendimiento en la capacidad de salto. Es importante considerar la altura de salto en la evaluación de futuros talentos deportivos ya que la mejora de los niveles de fuerza explosiva y de fuerza elástica son el potencial de base para mejorar las prestaciones del jugador en las acciones técnico-tácticas en las que el salto está presente (Bosco, 1992). En cuanto al peso corporal se evidenció que en las posiciones portero y extremos un mayor peso, estaba asociado a mayor masa adiposa. Esto queda reflejado en que el exceso de peso corporal se debe principalmente a los mayores valores de la masa adiposa no encontrándose de la misma manera en el caso de la masa muscular. Estas dos variables se constituyeron como las variables más significativas por sobre las demás y si bien las demás variables tenían una significancia en la capacidad de salto, no fue mayor a la encontrada en estas dos variables.

Por otro lado, dentro de las variables de la composición corporal, se pudo corroborar que la masa muscular fue la más significativa a la hora de favorecer la altura de salto alcanzada, obteniendo que por cada porciento (\%) de masa muscular la altura alcanzada aumentó en $0,95 \mathrm{~cm}$, por sobre la masa adiposa, la cual presentó una disminución del $0,87 \mathrm{~cm}$ de altura alcanzada por cada por ciento de masa adiposa aumentada.

Dentro de las variables antropométricas que se consideraban importantes en la capacidad de salto, no todas fueron significativas en este estudio. Las más relevantes fueron la variable peso y masa adiposa, las cuales presentan índices de correlación negativos $(p<0,05)$ en la altura alcanzada de todos los deportistas por posición de juego. El peso influyó negativamente con la altura de salto, es decir, cuando un sujeto tiene mayor peso asociado a masa adiposa, menor es la altura y velocidad alcanzada en pruebas de salto.

Otra variable que resultó significativa fue la masa muscular, la que al relacionarse con la capacidad de salto, resulta beneficiosa para poder lograr una mayor altura alcanzada. Esto permite a los jugadores de balonmano desarrollar mayores ventajas para su puesto específico. $\mathrm{Al}$ respecto de lo anteriormente expuesto, hacen referencia a los jugadores juveniles de balonmano de España, donde se evidenció que los jugadores más grandes y de mayor peso fueron los pivotes y porteros, siendo los centrales y extremos los que presentaron menores valores en esta variable (Vila et al.), algo que se corroboró con los resultados en este estudio.
Por lo tanto, el perfil antropométrico es de suma importancia para poder categorizar a los jugadores en un puesto determinado y según las funciones específicas. En concordancia con lo anterior, los resultados indican que los pivotes y porteros presentaron una menor capacidad de salto, una menor masa muscular, mayores índices de peso y una mayor masa adiposa. Considerando lo expuesto anteriormente, se debe señalar que el pivote se encuentra cerca del pórtico rival, por lo que no necesita una gran capacidad de salto, sin embargo, al encontrarse entre la defensa adversaria requiere mayor corpulencia para resistir las embestidas de los contrarios. Al respecto Correa de Sousa et al. (2011), en su estudio "características morfológicas de los atletas de balonmano masculino" señalan, que características como la estatura proporcionan una gran ventaja ofensiva, ya que el jugador puede lanzar el balón más fácilmente a la portería contraria, superando así a la defensa adversaria y también se presenta una ventaja defensiva, con un bloqueo alto.

Los porteros al no realizar mayores movimientos en el terreno de juego, no requieren ser los más livianos, esto explicaría de alguna forma su alto valor en la variable peso y la presencia de una menor masa muscular, y por ende en este estudio una mayor masa adiposa, que provoca que no posean una capacidad de salto relevante. En lo que respecta al somatotipo, estos sujetos muestran una tendencia a la meso-endomorfia, con un alto desarrollo de la masa muscular y con un alto índice de masa adiposa relativa.

En cuanto a otras posiciones en el juego se encuentran los primera línea y extremos, los que presentan valores semejantes, destacando una desarrollada capacidad de salto y un peso corporal menor en comparación a los jugadores de las posiciones portero y pivotes. En relación al somatotipo, se observa una tendencia a la Mesomorfia, es decir, que poseen una masa muscular predominante con una endomorfia y ectomorfia balanceada.

De lo expuesto anteriormente, se puede concluir que existen diferencias significativas entre las posiciones de juego y estas son inducidas por diversas variables, lo cual podría deberse a una especialización temprana en estos equipos, orientada por los procesos de selección que buscan la mejor eficiencia y rendimiento en este deporte que está adquiriendo gran relevancia en Chile. Los resultados obtenidos en este estudio coinciden con las referencias actuales en el perfil antropométrico, además de tener una coherencia con los datos de investigaciones realizadas en España y Portugal, en las cuales se muestra que los jugadores poseen características bastante similares a los sujetos de este estudio.

Además, considerando los resultados obtenidos y contrastándolos con los demás equipos evaluados, La Se- 
lección Chilena, seguida del Club Italiano de Villa Alemana, fueron los equipos que presentaron los mejores valores dentro de las variables del perfil antropométrico. En la selección Chilena se presentaron menores niveles de masa adiposa, mayores niveles de masa muscular y mayor altura alcanzada en el salto.

Respecto al somatotipo, se concluye que en el balonmano hay una predominancia de la Mesomorfia. Esto refiere a que los jugadores presentan una musculatura desarrollada, pero acompañada de un grado de adiposidad que repercute negativamente en la altura del salto alcanzado y en la velocidad de desplazamiento durante el juego. Es preciso señalar que este grupo de deportistas se encuentran en una etapa de maduración y especialización deportiva, la que al ser guiada a través de una correcta planificación del entrenamiento puede favorecer el rendimiento en este deporte.

Finalmente, se debe mencionar que este estudio fue considerado para fijar parámetros comparativos que po- drían resultar útiles como fundamento teórico en los procesos de selección deportiva que llevan a cabo los entrenadores o profesores de educación física en los clubes. Estos seleccionadores podrán tener una visión más específica respecto a las exigencias de cada posición en el campo de juego en términos de estructura corporal y capacidad de salto, que les permita orientar de mejor forma sus procesos de entrenamiento y por consiguiente obtener mejoras en el rendimiento deportivo.

\section{AGRADECIMIENTOS}

Agradecemos la participación y colaboración de los distintos equipos de balonmano de la región de Valparaíso y de la Selección Chilena así como a sus técnicos y jugadores, los cuales fueron el aporte principal a este trabajo. Indudablemente se debe agradecer, además, el esfuerzo y las ganas para que cada día este deporte se reconozca y masifique más en Chile.

BARRAZA, F.; YÁÑEZ, R.; TUESTA, M.; NUÑEZ, P.; ZAMORA, Y. \& ROSALES, G. Anthropometric profile in Chilean handball players according to playing position. Int. J. Morphol., 33(3):1093-1101, 2015.

SUMMARY: The primary purpose of this research was to determine the relationship between the anthropometric profile and jump capacity of male handball players from the Fifth Region of Chile and the National Handball Team and their specific position in the playing field. In the evaluation 74 handball players participated from the following teams: Club Italiano Villa Alemana, Club Italiano Quillota, Club Luterano, Club Viña del Mar, Club San José and the National Handball Team. Participants were in the cadet category aged 14 - 16. The results showed that within anthropometric variables, players from the Chilean Handball Team and the Club Italiano Villa Alemana are the closest to fulfilling all the criteria required to reach high competitive levels in this category. In reference to anthropometric variables, fat mass proved to be negatively related to jump height reached in all groups tested, while muscle mass presented significant correlation with the jump capacity in most of the teams. Regarding the somatotype, results obtained agree with the theoretical referents. These establish that handball player's somatotype is more likely to be mesomorph. The correlation indexes demonstrate that a lower fat mass percentage and a higher muscle mass percentage enhance the jump capacity in all playing positions. It is clear now that fat mass and muscle mass are important indicators when determining the jump capacity performance in players with similar characteristics of the ones in this study. This research is important in setting comparative parameters in the selection process and sport specialization regarding anthropometric characteristics and jump capacity according to specific position of handball players in the cadet category.

KEY WORDS: Anthropometric profile; Body composition; Somatotype; Jump capacity; Handball.

\section{REFERENCIAS BIBLIOGRÁFICAS}

Bayer, C. Técnica del Balonmano: la formación del jugador. Barcelona, Hispano Europea S.A., 1987.

Bosco, C. La valoración de la fuerza con el test de Bosco. Barcelona, Editorial Paidotribo, 1992.

Carter, J. E. L. \& Heath, B. H. Somatotyping - Development and applications. Cambridge, Cambridge University Press, 1990.
Carter, J. E. The Heath-Carter Somatotype Method. San Diego, San Diego State University Syllabus Service, 2002.

Correa de Sousa, J. C.; de Miranda, E. F.; Alves de Moraes Filho, J.; Ribeiro, D. B. G.; Tonello, L.; de Souza, M. A. F.; \& Dantas, E. H. M. Características morfológicas de los atletas de balonmano masculino. Análisis comparativo con los mejores atletas de la categoría juvenil. Mot. Hum., 12(1):16-21, 2011. 
Drinkwater, D. T. An anatomically deroved method for the anthropometric estimation of human body composition. Ph.D. Thesis. British Columbia, Simon Fraser University, 1984.

Ekblom, B. Applied physiology of soccer. Sports Med. 3(1):5060, 1986.

Esparza, R. F. Manual de Cineantropometría. Pamplona, FEMEDE, 1993.

Fernández, R. J. J. Estructura condicional en los preseleccionados gallegos de diferentes categorías de formación en balonmano. Tesis Doctoral. La Coruña, Universidad de La Coruña, 2000.

Gorostiaga, E. M.; Izquierdo, M.; Iturralde, P.; Ruesta, M. \& Ibáñez, $\mathrm{J}$. Effects of heavy resistance training on maximal and explosive force production, endurance and serum hormones in adolescent handball players. Eur. J. Appl. Physiol. Occup. Physiol., 80(5):485-93, 1999.

Kerr, D. A. An anthropometric method for the fractionation of skin, adipose, muscle, bone and residual tissue masses in males and females age 6 to 77 years. M.Sc. Thesis. British Columbia, Simon Fraser University, 1988.

Moreno, F. Balonmano: Detección, Selección y Rendimiento de Talentos. Madrid, Gymnos, 2004.

Malina, R. M. \& Bouchard, C. Growth, Maduration and Physical Activity. Champaign, Human Kinetics, 1991.

Norton, K. \& Olds, T. Antropométrica. Sydney, University of New South Wales Press, 1996.

Ramírez, F. E.; Iglesias, P. M. C. \& Álvarez, P. Estudio de las características antropométricas de los jugadores cadetes de balonmano de Portugal. Efdeportes.com/Revista Digital, 11(101), 2006

Srhoj, V.; Marinovic, M. \& Rogulj, N. Position specific morphological characteristics of top-level male handball players. Coll. Antropol., 26(1):219-27, 2002.

Van Muijen, A. E.; Joris, H.; Kemper, H. C. G. \& Schenau, G. J. V. I. Throwing practice with different ball weights: Effects on throwing velocity and muscle strength in female handball players. Sports Med. Train. Rehabil., 2(2):103-13, 1991.

Vila, S. H.; Abraldes, V. J. A. \& Rodriguez, S. N. Estudio del perfil antropométrico del jugador juvenil de balonmano en la Región de Murcia. Retos. Nuevas Tend. Educ. Fis. Deporte Recreac., 16(2):80-5, 2009.

\author{
Dirección para Correspondencia: \\ Prof. Fernando Barraza Gómez \\ Director Académico \\ Magister en Evaluación y Planificación \\ del Entrenamiento Deportivo \\ Carrera de Educación Física \\ Universidad Viña del Mar \\ Viña del Mar \\ CHILE
}

Email: fbarraza@uvm.cl

Recibido: 29-08-2014

Aceptado: 14-07-2015 